\title{
SNP Discovery for mapping alien introgressions in wheat
}

\author{
Vijay K Tiwari ${ }^{1}$, Shichen Wang ${ }^{2}$, Sunish Sehgal', Jan Vrána ${ }^{3}$, Bernd Friebe ${ }^{1}$, Marie Kubaláková ${ }^{3}$, Praveen Chhuneja ${ }^{4}$,
} Jaroslav Doležel ${ }^{3}$, Eduard Akhunov², Bhanu Kalia', Jamal Sabir ${ }^{5}$ and Bikram S Gill ${ }^{1,5^{*}}$

\begin{abstract}
Background: Monitoring alien introgressions in crop plants is difficult due to the lack of genetic and molecular mapping information on the wild crop relatives. The tertiary gene pool of wheat is a very important source of genetic variability for wheat improvement against biotic and abiotic stresses. By exploring the $5 \mathrm{M}^{9}$ short arm $\left(5 \mathrm{M}^{\mathrm{g}} \mathrm{S}\right)$ of Aegilops geniculata, we can apply chromosome genomics for the discovery of SNP markers and their use for monitoring alien introgressions in wheat (Triticum aestivum $\mathrm{L}$ ).

Results: The short arm of chromosome $5 M^{9}$ of Ae. geniculata Roth (syn. Ae. ovata L.; $2 n=4 x=28, U^{9} U^{9} M^{9} M^{9}$ ) was flow-sorted from a wheat line in which it is maintained as a telocentric chromosome. DNA of the sorted arm was amplified and sequenced using an Illumina Hiseq 2000 with 45x coverage. The sequence data was used for SNP discovery against wheat homoeologous group-5 assemblies. A total of 2,178 unique, 5M95-specific SNPs were discovered. Randomly selected samples of $595 \mathrm{M}^{9} \mathrm{~S}$-specific SNPs were tested (44 by KASPar assay and 15 by Sanger sequencing) and $84 \%$ were validated. Of the selected SNPs, $97 \%$ mapped to a chromosome $5 \mathrm{M}^{9}$ addition to wheat (the source of $\mathrm{t}^{\mathrm{M}} \mathrm{M}^{9} \mathrm{~S}$ ), and $94 \%$ to $5 \mathrm{M}^{9}$ introgressed from a different accession of Ae. geniculata substituting for chromosome 5D of wheat. The validated SNPs also identified chromosome segments of 5M ${ }^{9} \mathrm{~S}$ origin in a set of T5D-5M ${ }^{9}$ translocation lines; eight SNPs (25\%) mapped to TA5601 [T5DL·5DS-5M9S(0.75)] and three (8\%) to TA5602 [T5DL. 5DS-5M9S (0.95)]. SNPs (gsnp_5ms83 and gsnp_5ms94), tagging chromosome T5DL·5DS-5M9S(0.95) with the smallest introgression carrying resistance to leaf rust ( $(r 57)$ and stripe rust (Y $r 40)$, were validated in two released germplasm lines with $L$ r57 and Yr40 genes.
\end{abstract}

Conclusion: This approach should be widely applicable for the identification of species/genome-specific SNPs. The development of a large number of SNP markers will facilitate the precise introgression and monitoring of alien segments in crop breeding programs and further enable mapping and cloning novel genes from the wild relatives of crop plants.

\section{Background}

Crop plants have a narrow genetic base because of domestication and breeding. Moreover, many crop plants have gone through a polyploidization bottleneck. Introgressive hybridization with wild relatives, often described as alien introgression, is widely used to broaden the crop genetic base. Bread or hexaploid wheat (Triticum aestivum L., $2 \mathrm{n}=6 \mathrm{x}=42$, AABBDD genome), which accounts for $95 \%$ of the harvested wheat crop, traces its origin to a rare hybridization event $\sim 6,000$ years ago

\footnotetext{
* Correspondence: bsgill@ksu.edu

'Wheat Genetics Resource Center, Department of Plant Pathology, Kansas

State University, Manhattan, KS 66506, USA

${ }^{5}$ Department of Biological Sciences, Faculty of Sciences, King Abdulaziz

University, Zeddah, Saudi Arabia

Full list of author information is available at the end of the article
}

involving T. turgidum L. $(2 \mathrm{n}=4 \mathrm{x}=28$, AABB $)$ and Aegilops tauschii Coss. $(2 \mathrm{n}=2 \mathrm{x}=14$, DD) [1-3]. McFadden and Sears [1], Kihara [2], and McFadden and Sears [3] have reproduced this hybridization event to generate 'synthetic wheat'. These two species, and wheat landraces, constitute the primary gene pool of wheat [4]. Synthetic wheats and direct crosses between $T$. aestivum and Ae. tauschii $[5,6]$ have been used to enrich the bread wheat genetic base. Extensive wheat genetic resources and marker systems [7-9] are transferable and can be used for mapping of alien introgressions [10], characterizing genetic diversity [11] and gene isolation $[12,13]$ from the primary gene pool.

Tetraploid, emmer wheat T. turgidum L. arose $\sim 350,000$ years ago from a hybridization between $T$. urartu Tuanian

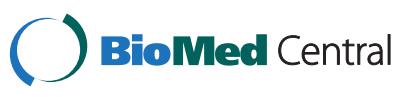


ex Gandilyan $(2 \mathrm{n}=2 \mathrm{x}=14, \mathrm{AA})$ and a B-genome species, whose closest living relative is Ae. speltoides Tausch $(2 \mathrm{n}=2 \mathrm{x}=\mathrm{SS})$ [14-16]. These two species, together with the A-genome species T. monococcum L. subsps. monococcum and aegilopoides, the tetraploid wheat sibling species $T$. timopheevii Zhuk. ( $2 \mathrm{n}=4 \mathrm{x}=28$, AAGG) and D genome cluster present in polyploid Aegilops species [17] constitute the secondary gene pool. Usually, wheat marker systems can be used to map alien introgressions from secondary gene pool.

Hundreds of other species in the Triticeae tribe contain genomes other than A, B and D, and these species constitute the tertiary gene pool of bread wheat. All of these species can be hybridized with hexaploid wheat to produce amphiploids, addition and translocation lines [18-20]. Chromosome engineering approaches have been used [4,21-24] to produce small alien transfers without linkage drag. Cytological approaches have been extensively used to identify alien introgression lines. However, these approaches lack throughput and resolution and are not suitable when analyzing a very large number of progeny for detecting a rare recombination event [25].

Molecular markers can detect small chromosome segments not detectable cytologically and permit easier identification of the introgressed alien fragments. Microsatellite markers have been used extensively in the primary and secondary gene pools, however, they have low transferability to tertiary gene pool species, and the lack of locus specificity hampers their application [26]. Wheat EST bin maps have been explored as a source of markers, but polymorphic markers are rare [4]. Single-nucleotide polymorphism (SNP) markers have become the technology of choice for all organisms because of their wide distribution in genomes and compatibility with high, multiplex detection systems [27-31]. Advances in SNP marker development in wheat and the availability of various SNP genotyping platforms now permit highthroughput and cost-effective genotyping [27,28,31].

Despite the progress in DNA marker technology, mapping large and polyploid genomes such as wheat remains a daunting task. Mapping and sequencing complex plant genomes can be simplified by dissecting the chromosomes by flow cytometric sorting [32]. This approach reduces sample complexity and enables analysis at the subgenomic level. Flow cytometric chromosome sorting has been implemented successfully in many plant species, including cultivated cereals (such as bread and durum wheat), barley, rye, oats, rice and maize [33]. Recently, Molnár et al. reported flow-sorting of individual chromosomes from Ae. umbellulata Zhuk. $(2 \mathrm{n}=2 \mathrm{x}=14$, UU) and Ae. comosa Sm. In Sibth. \& Sm. $(2 \mathrm{n}=2 \mathrm{x}=14$, $\mathrm{MM}$ ) and from their natural allotetraploid hybrids (Ae. biuncialis Vis. and Ae. geniculata) [34,35]. This study provided opportunity for the next-generation sequencing of individual Aegilops chromosomes for the development of sequence-based markers and their application in wheat breeding.

Ae. geniculata, also called ovate goatgrass, is found widely distributed in the Middle East. A member of the tertiary gene pool of wheat, Ae. geniculata arose from hybridization between the diploid species Ae. umbellulata and Ae comosa [36,37] and is an important source of useful genes for wheat improvement [20,38-41]. The Ae. geniculata genome has been introgressed into wheat, and single-chromosome, addition lines were developed by Friebe et al. [42]. Previously, we have reported on the transfer of genes Lr57 and Yr40 [43] and Sr53 [44] from chromosome $5 \mathrm{M}^{\mathrm{g}}$ of Ae. geniculata to chromosome 5D of wheat. In this study, we present the first report on alien chromosome-based SNP discovery and its application in mapping of alien introgression in wheat.

\section{Results}

Flow-sorting and sequencing of the $5 \mathrm{M}^{\mathrm{g}}$ short arm

The analysis of DAPI-stained, chromosome suspensions prepared from a wheat-Ae. geniculata $\mathrm{t}^{\mathrm{g}} \mathrm{M}^{\mathrm{g} S}$ telocentric addition line resulted in histograms with five peaks of fluorescence intensity (flow karyotypes) (Figure 1). The leftmost peak represents telochromosome $t 5 \mathrm{M}^{\mathrm{g}} \mathrm{S}$, which was well resolved from composite peaks I, II, III and peak

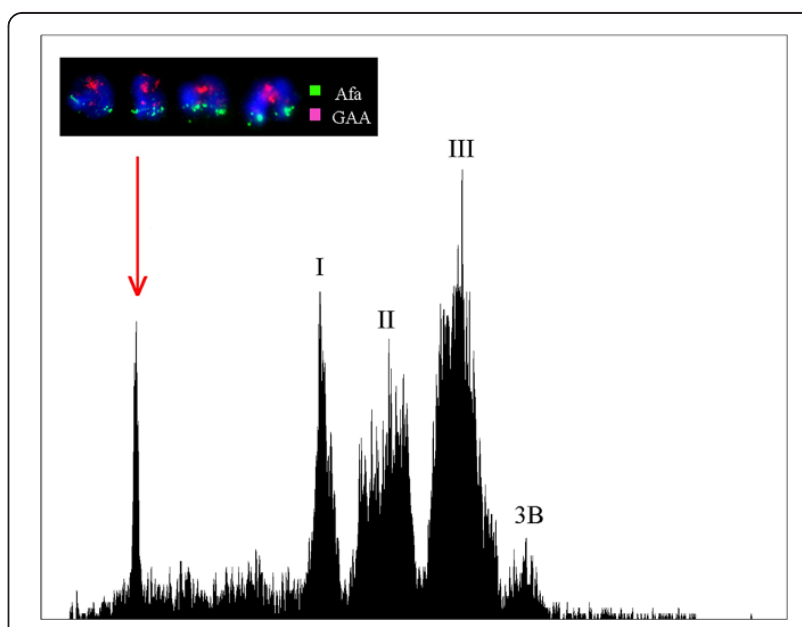

Figure 1 Flow cytometric analysis of $5 \mathrm{M}^{\mathbf{9}}$ short arm. Histogram of the relative fluorescence intensity (flow karyotype) obtained after the analysis of a DAPI-stained chromosome suspension prepared from a wheat-Ae. geniculata ditelosomic addition line $t 5 \mathrm{M}^{9} \mathrm{~S}$. The leftmost peak represents chromosome $t 5 \mathrm{M}^{9} \mathrm{~S}$. In addition, the flow karyotype comprises three composite peaks I, II and III, representing groups of wheat chromosomes, and a peak representing wheat chromosome 3B. Inset: Examples of flow sorted chromosomes after FISH with probes for the Afa-family (yellow-green) and [GAA]n repeats (red). The chromosomes were counterstained by DAPI (blue). $X$ axis: DAPI fluorescence intensity; $Y$ axis: number of events. 
$3 \mathrm{~B}$ of the bread wheat chromosomes; $\mathrm{t}^{\mathrm{g}} \mathrm{M}^{\mathrm{g}} \mathrm{S}$ was flowsorted with $92.6 \%$ purity. A random mix of chromosome and chromatid fragments contaminated the sorted fractions. DNA amplified from flow-sorted $t 5 \mathrm{M}^{\mathrm{g}} \mathrm{S}$ was sequenced by the Illumina technology. In total, we generated more than 153 million reads of $100 \mathrm{bp}$ from one-lane HiSeq sequencing. After quality trimming and filtering, about 145 million reads ( 95\%) were used for mapping, providing approximately $\sim 45 \mathrm{x}$ coverage for t5 $\mathrm{M}^{\mathrm{g}} \mathrm{S}$. De novo assembly of the $5 \mathrm{M}^{\mathrm{g}} \mathrm{S}$ reads resulted in 7,319 contigs with length $\geq 500 \mathrm{bp}$ and average coverage depth of 20x (Additional file 1: Figure S1). Blast against wheat EST sequences showed that 1,408 of the 7,319 contigs may contain genes. Sequence data generated in this study was submitted to SRA database (accession: SRX474187).

\section{Mapping of reads and SNP calling}

For mapping the $5 \mathrm{M}^{\mathrm{g} S}$ reads, we used 5AS, 5BS and 5DS chromosome shotgun sequence assemblies provided by the International Wheat Genome Sequencing Consortium. A maximum of three mismatches were allowed for each read for mapping (Additional file 2: Figure S2) on reference group of chromosome five short arm assemblies (5AS, 5BS and 5DS). Depending on the references used to map the reads, only about $30 \%, 23 \%$ and $25 \%$ of reads could be mapped to the $5 \mathrm{AS}, 5 \mathrm{BS}$ and $5 \mathrm{DS}$ assemblies, respectively. The mapped $5 \mathrm{M}^{\mathrm{g} S}$ reads covered $103,203,161$ (52\%) of the $5 \mathrm{AS}, 93,086,474$ (53\%) of the $5 \mathrm{BS}$ and $69,678,915(47 \%)$ of the 5DS assemblies (Additional file 2: Figure S2). Based on the alignments of the $5 \mathrm{M}^{\mathrm{g}} \mathrm{S}$ reads, we discovered 976,754 5AS, 675,007 5BS and 851,722 5DS raw variations. After filtering with a coverage depth of 4 and a SNP quality of 50; 277770 (5AS), 203522 (5BS) and 355765 (5DS) high-quality SNPs were retained, which were used for further analysis after analyzing 220624667 base pair sequences in total (Table 1). SNP densities of the $5 \mathrm{M}^{\mathrm{g}} \mathrm{S}$ sequences against $5 \mathrm{AS}, 5 \mathrm{BS}$ and 5DS were observed to be $1.3,0.9$ and $1.6 \mathrm{SNPs} / \mathrm{kb}$, respectively, with an average of $1.3 \mathrm{SNP} / \mathrm{kb}$. We searched against the wheat EST and NCBI nr databases with blastn and blastx and discovered 35749 (5AS), 31526

\begin{tabular}{|c|c|c|c|c|}
\hline $\begin{array}{l}\text { Reference } \\
\text { assemblies }\end{array}$ & Mapped reads & Raw SNPs & Filtered SNPs & $\overline{\text { Genic SNPs }}$ \\
\hline $5 A S$ & $45,989,152$ & 976,754 & 277,770 & 35,749 \\
\hline $5 \mathrm{BS}$ & $35,168,202$ & 675,007 & 203,522 & 31,526 \\
\hline 5DS & $38,012,773$ & 851,722 & 355,765 & 11,704 \\
\hline
\end{tabular}

(5BS) and 11704 (5DS) SNPs located in the gene coding regions.

\section{$5 \mathrm{M}^{\mathrm{g}}$-genome-specific SNPs}

After mapping the $5 \mathrm{M}^{\mathrm{g}} \mathrm{S}$ reads from Ae. geniculata on the group-5, short-arm assemblies of Chinese Spring, we focused on finding $5 \mathrm{M}^{\mathrm{g}}$-specific SNPs. SNPs that had the same alleles on $5 \mathrm{~A}, 5 \mathrm{~B}$ and $5 \mathrm{D}$ but a different allele on $5 \mathrm{M}^{\mathrm{g}}$ were identified. It was found that 2,178 SNPs have the same alleles in the 5AS, 5BS and 5DS contigs and different alleles in the $5 \mathrm{M}^{\mathrm{g}} \mathrm{S}$ sequences. These SNPs were putative $5 \mathrm{M}^{\mathrm{g}}$-specific SNPs (for convenience, we only kept the SNP positions belonging to 5AS contigs). To make sure that the flanking sequences of $5 \mathrm{M}^{\mathrm{g} S} \mathrm{~S}$-specific SNPs matched the $5 \mathrm{M}^{\mathrm{g}} \mathrm{S}$ assemblies, flanking sequences of $5 \mathrm{M}^{\mathrm{g}} \mathrm{S}$-specific SNPs were blasted to the $5 \mathrm{M}^{\mathrm{g}} \mathrm{S}$ assemblies so that the primers designed based on the flanking sequences would work for both $5 \mathrm{~A}(5 \mathrm{~B}, 5 \mathrm{D})$ and $5 \mathrm{M}^{\mathrm{g}}$. To eliminate interference from variations that may locate in the flanking sequences, only 104 SNPs that showed no variation in the 100-bp flanking sequences of SNPs between the $5 \mathrm{~A}$ and $5 \mathrm{M}^{\mathrm{g}} \mathrm{S}$ contigs were selected (Additional file 3: Table S1).

\section{SNP validation}

In order to analyze the authenticity of the discovered SNPs, 44 sequences with one SNP each were randomly chosen from SNP sequences and used to design a KASPar genotyping assay. Of the 44 KASPar genotyping assays, six SNPs had identical alleles in wheat and $A e$. geniculata and two SNPs showed heterozygous alleles in Ae. geniculata accession TA2899. On average, 84\% SNPs were validated in Chinese Spring and the Ae. geniculata TA2899 (Figure 2a).

For Sanger sequencing-based validation, we tested 15 primers derived from SNP sequences; three did not show any amplification in either line suggesting the need for PCR optimization. Of 12 primers tested between Chinese Spring and Ae. geniculata TA2899, the SNP validation rate was $\sim 84 \%$, which was similar to the results from the KASPar assay.

KASPar assays were done on wheat cultivars Chinese Spring, WL711 and seven Ae. geniculata accessions to test the applicability of the 44 candidate SNPs (Table 2). Sixty-nine to $85 \%$ of the SNPs showed different alleles between wheat and the Ae. geniculata lines (Table 2). Approximately $10 \%$ of the SNPs were polymorphic between Ae. geniculata accessions TA1800 and TA10437, and polymorphic SNPs were tested on a subset of an $F_{2}$ population (36 lines) developed from a cross between TA1800 and TA10437 (Figure 2d). Of a total of 59 SNPs tested, 33 belonged to genic sequences and 26 belonged to non-genic sequences. SNP validation rates were similar for both genic (86\%) and non-genic sequences. 


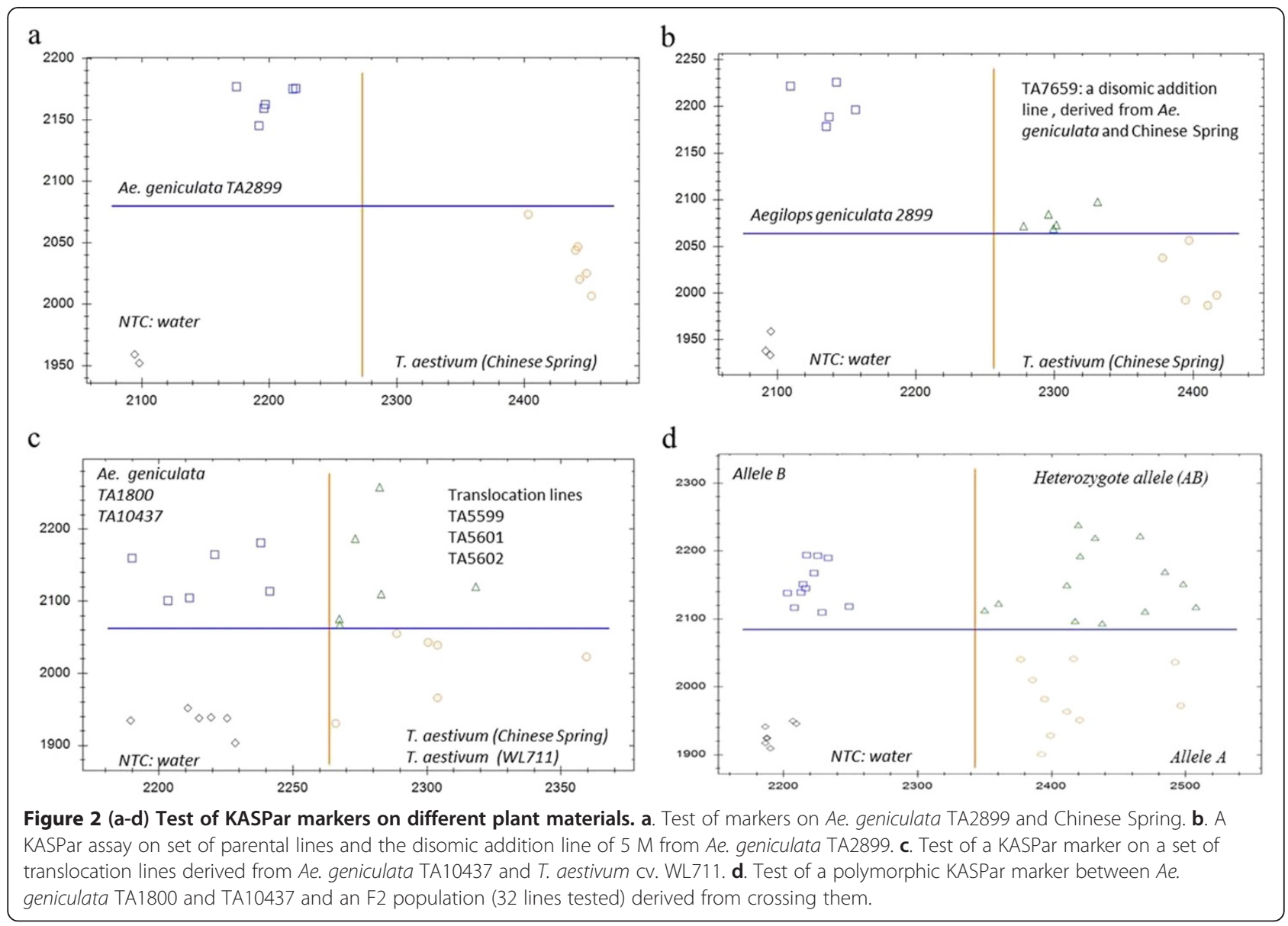

\section{Application of $5 \mathrm{M}^{\mathrm{g}}$-specific SNPs \\ Addition lines}

Validated SNPs between Chinese Spring and Ae. geniculata accession TA2899 were used to identify $5 \mathrm{M}^{\mathrm{g}}$ chromosome in the chromosome complement of Chinese Spring wheat. In the $5 \mathrm{M}^{\mathrm{g}}$ addition line, $5 \mathrm{M}^{\mathrm{g}}$-specific SNPs were expected to have heterozygous condition, because wheat chromosomes $5 \mathrm{~A}, 5 \mathrm{~B}$ and $5 \mathrm{D}$ carry the alternative allele. Out of 37 SNPs, 97\% detected heterozygous alleles (Figure 2b), confirming the presence of both wheat and Ae. geniculata chromosomes in the tested addition line (TA7659).

\section{Substitution and translocation lines}

Out of 44 SNPs tested on T. aestivum cv. WL77 and Ae. geniculata TA10437, 36 SNPs were validated and showed heterozygous alleles in alien translocation lines TA6675 and TA5599 (Figure 3b and c). Nine of 36 SNPs (25\%) had a heterozygous allele in TA5601 (Figure 3d), and three SNPs $(8.3 \%)$ were present (Figure 3e) in the translocation line TA5602.

Two germplasm lines (KS11WGGRC53-J and KS11W GGRC53-O) were developed using TA5602. Of the three SNPs (gsnp_5ms62, gsnp_5ms83 and gsnp_5ms94) validated in terminal translocation line TA5602 (Figure 3e), two (gsnp_5ms83 and gsnp_5ms94) mapped in KS11WG GRC53-J (Figure 3f). Only one SNP (gsnp_5ms94) mapped in germplasm line KS11WGGRC53-O (Figure 3g).

\section{Discussion}

The gene pools with the wild relatives of crops hold tremendous potential for crop improvement for resistance to biotic and abiotic stresses. In wheat, many alien

Table 2 Validation percentage of $445 \mathrm{M}^{\mathrm{g}} \mathrm{S}$-specific SNPs on a set of seven Ae. geniculata lines against two wheat cultivars (T. aestivum CV. Chinese Spring and WL711)

\begin{tabular}{|c|c|c|c|c|c|c|c|}
\hline \multirow{2}{*}{$\begin{array}{l}\text { Wheat } \\
\text { cultivars }\end{array}$} & \multicolumn{7}{|c|}{ Validation of $5 \mathrm{M}^{9} \mathrm{~S}$-specific SNPs in selected Ae. geniculata accessions (\%) } \\
\hline & TA2899 & TA10437 & TA1800 & TA1801 & TA2786 & TA2041 & TA10029 \\
\hline Chinese spring & 85.4 & 83.7 & 83.3 & 78.4 & 78.8 & 69.4 & 68.8 \\
\hline WL711 & 75.0 & 81.4 & 81.4 & 78.8 & 76.5 & 73.0 & 65.7 \\
\hline
\end{tabular}




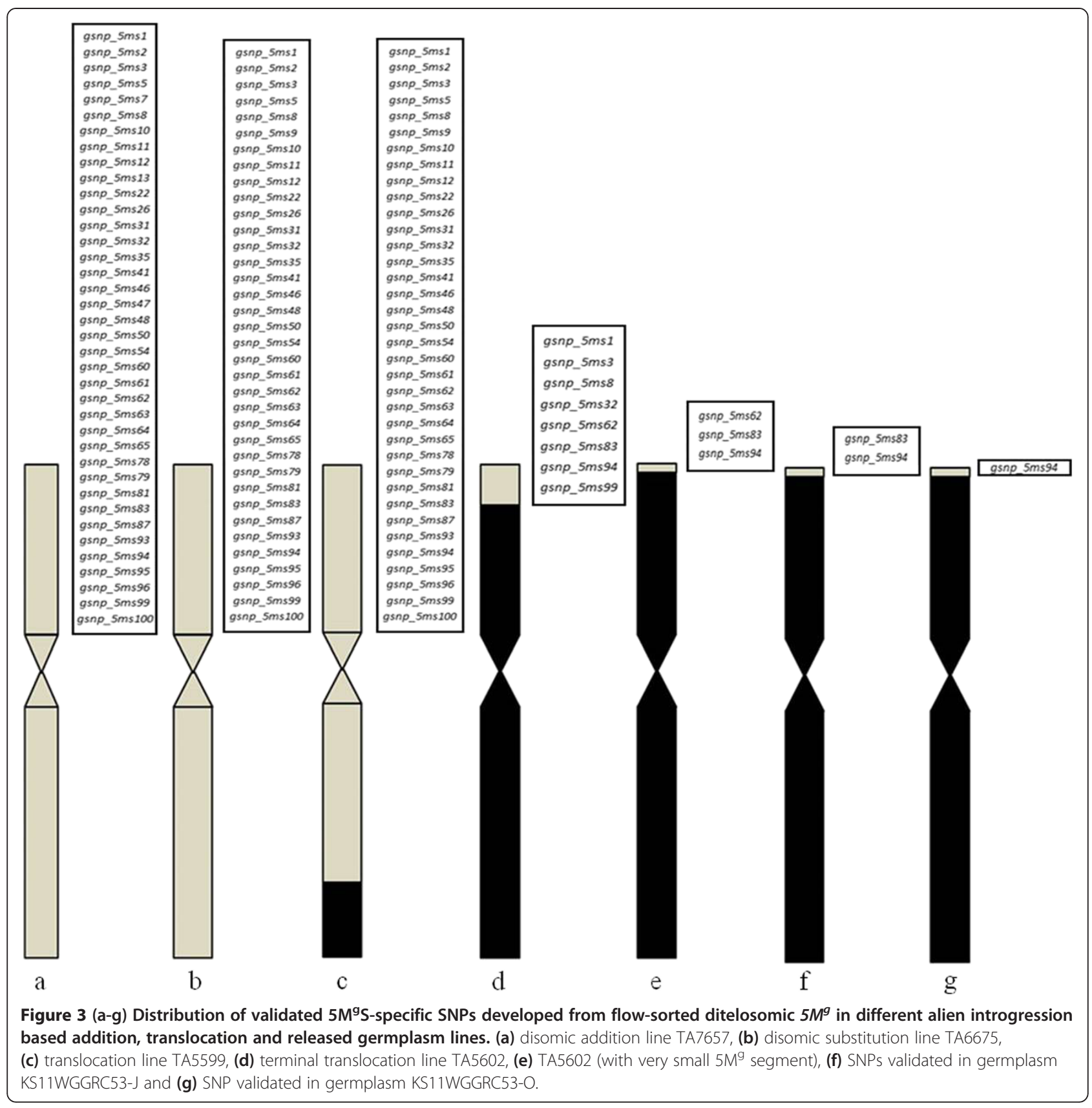

introgression and translocation lines involving members from tertiary gene pool have been produced [20]. Exploiting alien germplasm has been slow because of the time-consuming process of interspecific hybridization and isolation of addition and translocation lines with reduced linkage drag [19,20]. A number of approaches are available to minimize alien chromatin for reducing linkage drag $[20,21,45-49]$; the most widely used approach is to induce meiotic recombination between alien chromosome and its homoeologous (partially homologous) wheat chromosome. However, the frequency of homoeologous recombination between wheat and alien chromosomes is low
( $\sim 3 \%)$, and a large number of progeny must be screened to isolate recombinants with small alien transfers [50]. Cytological methods, such as chromosome banding, genomic in situ hybridization (GISH) and fluorescent in situ hybridization (FISH), have been used extensively to identify introgression and translocation lines in wheat [22,51-53], but these approaches are low throughput and have resolution limitations [25,43]. Molecular markers have been used to identify alien fragments; but limited availability of alien, chromosome-specific molecular markers, especially for the tertiary gene pool species, hampers the characterization of useful lines with traits of interest 
[50]. As an example, only a small number of U- and Mgenome-specific SSR markers are available, greatly limiting marker-assisted selection of wheat-Ae. geniculata and wheat-Ae. biuncialis introgression lines [54-56]. Practically no non-radioactive markers were available for the analysis of the wheat-Ae. geniculata introgressions described herein, and all previous mapping was done using RFLP analysis [43]. Qi et al. [9] used EST-STS markers to identify alien introgression lines, but polymorphism was very low and only three or fewer polymorphic markers were discovered for each arm [9]. Because the selection was based on only a few markers, useful smaller translocation may have escaped detection. Therefore, the development of a system for a user-friendly high-throughput method of identifying alien chromosome(s)-based introgression and translocation lines as reported here is highly significant.

Flow cytometric chromosome sorting has been a foundation of the genomics of the Triticeae tribe [57-60]. Molnár et al. [34] applied the flow-sorting technique on the allotetraploid species Ae. biuncialis and Ae. geniculata and their diploid progenitors Ae. umbellulata and Ae. comosa $[34,35]$. Their results provided an opportunity for the molecular analysis of wild Aegilops chromosomes and developing Aegilops chromosome-specific markers. To date, only a few studies have reported on the development of U- and M-genome-specific SSR markers [34,35,50]. In this study, we flow-sorted a ditelosomic addition wheatAe. geniculata line to isolate the short arm of the Ae. geniculata $5 \mathrm{M}^{\mathrm{g}}$ chromosome with $\sim 95 \%$ purity. This approach reduced DNA sample complexity and permitted the development of markers specific for the short arm of $5 \mathrm{M}^{\mathrm{g}}$ (Figure 1). Approximately 145 million reads ( 95\%) of total sequence were obtained for mapping after trimming the data $(\sim 45 \mathrm{X})$, and the deep sequencing data was used for SNP discovery.

SNP discovery using next generation sequencing (NGS) was successfully used in small-genome plants, such as Arabidopsis and rice, because their reference genomes are available $[61,62]$. SNP discovery in complex genomes without a reference genome, such as wheat $[28,63]$ and barley $[64,65]$, can be achieved through NGS. However, the lack of accurate reference genome sequences can create ambiguities in SNP calling, which can be further complicated by the presence of paralogs and DNA repeats [66]. We used stringent mapping parameters to minimize erroneous base calling and misaligned reads. Our SNP discovery was greatly assisted by the availability of $5 \mathrm{AS}, 5 \mathrm{BS}$ and 5DS chromosome shotgun sequences and assemblies developed under the International Wheat Genome Sequencing Consortium Survey Sequencing Initiative. Only about $30 \%, 23 \%$ and $25 \%$ of $5 \mathrm{M}^{\mathrm{g}}$ short arm reads could be mapped to the 5AS, 5BS and 5DS assemblies, respectively, due to the low coverage of $5 \mathrm{M}^{\mathrm{g}} \mathrm{S}$ sequences on reference assemblies, small reads and probably the diversity of the sequences. Our SNP-filtering criterion was similar to that in previously published reports $[67,68]$ and yielded 277,770 (5AS), 203,522 (5BS) and 355,765 (5DS) highquality SNPs (Table 1). In this research we generated single end sequencing reads, assembled data provided us $\sim 7000$ contigs $>=500$ bp. Blast against wheat EST database of these larger contigs suggested $\sim 1400$ contigs with genes on Ae. geniculata chromosome arm $5 \mathrm{M}^{\mathrm{g}} \mathrm{S}$.

The estimated SNP frequency in our study was $~ 1.3$ $\mathrm{SNPs} / \mathrm{kb}$ of the total analyzed sequences. This frequency is slightly lower than those of previous reports; Trick et al. [67] found an average density of $1.80( \pm 1.46) \mathrm{SNP} / \mathrm{kb}$ and Ravel et al. [68] estimated SNP frequency to be 2.99 SNP/kb [68]. The lower estimated SNP frequency can be attributed to the low coverage of $5 \mathrm{M}^{\mathrm{g}}$ sequences on reference assemblies and a stringent filtering criterion. For developing M-genome-specific SNPs, we needed identical SNP alleles in 5AS, 5BS and 5DS but different in $5 \mathrm{M}^{\mathrm{g}} \mathrm{S}$. We aligned the $100-\mathrm{bp}$ flanking sequences of the SNPs that were discovered based on the three references (5AS, 5BS and 5DS contigs) and only those sequences were selected that showed $100 \%$ similarity on the flanking sequences of the SNP. Critical selection of SNPs against 5AS, 5BS and 5DS yielded 2,178 reliable $5 \mathrm{M}^{\mathrm{g}}$-specific SNPs which were around $1 \%$ of total SNPs discovered.

Our study identified $\sim 2,178$ chromosome $5 \mathrm{M}^{\mathrm{g}} \mathrm{S}$-specific SNPs, providing a quick approach for developing markers that would facilitate identifying alien addition and translocation lines. For M-genome-specific marker development, we shortlisted 104 sequences with unique SNPs. Using two different SNP validation approaches, we tested 59 randomly selected SNPs in wheat cultivar Chinese Spring and Ae. geniculata TA2899, and the validation rate was found to be $\sim 84 \%$. We tested Chinese Spring, WL711, and seven Ae. geniculata accessions. When comparing Chinese Spring against all the Ae. geniculata accessions, the average validation was $78.4 \%$, with a range of $68.8 \%$ to $85.4 \%$. For another set involving wheat line WL711 and the seven Ae. geniculata lines, the average validation rate was $76.0 \%$, with a range of $65.7 \%$ to $81.4 \%$. These results indicate the fixation of more than $70 \%$ of $5 \mathrm{M}^{\mathrm{g}}$-specific alleles in Ae. geniculata accessions (TA1800, TA1801, TA2847, TA2899, TA10029, TA2041 and TA10437), suggesting the usefulness of these SNPs in multiple Ae. geniculata accessions. We compared the validation rates of 59 SNPs (33 genic and 26 non-genic sequences). The validation rate for the genic and non-genic SNPs was very similar ( $86.1 \%$ and $85.2 \%$, respectively). The validated $5 \mathrm{M}^{\mathrm{g}} \mathrm{S}$-specific markers (Additional file 3: Table S1) will be useful for monitoring introgression $\left(5 \mathrm{M}^{\mathrm{g}} \mathrm{S}\right)$ in $A$ e. comosa, Ae. geniculata and Ae. biuncialis, because they share a common $\mathrm{M}$ genome. The KASPar assay used in this study provides a cheap and high-throughput means for 
identifying alien introgressions because one assay mix is sufficient for $\sim 2,500$ reactions. Validated SNPs between Chinese Spring and Ae. geniculata TA2899 were used to identify an alien disomic addition line (TA7659). Approximately $97.3 \%$ of the validated SNPs identified the addition line with heterozygous alleles (Figure 2b), confirming the presence of both wheat and Ae. geniculata chromosomes in TA7659. Forty-four $5 \mathrm{M}^{\mathrm{g}} \mathrm{S}$-specific SNPs also were tested on WL711, the Ae. geniculata accession TA10437 and a set of their substitution and translocation lines. As expected 94.4\%, 94.4\%, 25\%, and 8.3\% of the validated SNPs showed heterozygous alleles in TA6675, TA5599, TA5601 short arm and TA5602, respectively. These data clearly indicate the applicability of SNPbased identification for alien addition, substitution and translocation lines.

We also validated two released germplasm lines (KW11wggrc53-J and KS11wggrc53-O) developed by crossing TA5602 with two susceptible winter wheat lines Jagger and Overley. Both lines were found to have $5 \mathrm{M}^{\mathrm{g} S}$-specific SNPs mapped on TA5602 suggesting that two markers (gsnp_5ms83 and gsnp_5ms94) could be used for marker-assisted selection for disease resistance genes ( Lr57 and Yr40).

\section{Conclusions}

To conclude, this study marks an important step forward for utilizing wild and related resources of wheat. For the first time, an arm of an Aegilops chromosome from the tertiary gene pool of wheat was successfully flow-sorted and sequenced by Illumina technology. Recently it has been established that using 'Fluorescence in situ hybridization in suspension' approach (FISHIS) individual Aegilops chromosomes can be flow-sorted with high purity from wheat-Aegilops disomic addition and substitution lines (Dolezel et al. personal communication). Alternatively, advances in flow cytogenetics have made possible even the flow-sorting of Aegilops chromosomes from respective Aegilops species [35]. The approach reported in this article can be used for marker development from targeted flow-sorted Aegilops chromosome(s) and their applications in marker assisted selection (Figure 4). Next-generation sequencing offers a cheap way to develop sequence-based markers for molecular analysis of Aegilops chromosomes. The ability to purify chromosome arms of Aegilops species will be very useful for physical mapping, constructing arm-specific BAC libraries and developing NGS-based genic and low-copy sequences to make chromosome- and genome-specific markers. Recently released flow-sorted chromosome arm based survey sequenced assemblies of all wheat chromosomes will be very useful in alien chromosome based genome specific SNP discovery (https://urgi.versailles.inra.fr/download/iwgsc/). Mapped arm- and genome-specific SNP markers can be used to identify alien chromosome segments with a gene of interest for pre-breeding in wheat improvement programs.

\section{Methods}

Plant material

All plant materials used in this study were procured, developed and maintained by the Wheat Genetics Resource Center at Kansas State University (http://www.k-state.edu/ wgrc/). Plant materials include Ae. geniculata (accession TA2899) $5 \mathrm{M}^{\mathrm{g}}$ chromosome disomic addition line (TA7659) in wheat cultivar Chinese Spring [42], a disomic substitution line [TA6675; DS5M $\left.\mathrm{M}^{\mathrm{g}}(5 \mathrm{D})\right]$, and three wheat-Ae. geniculata translocation lines TA5599, TA5601, and TA5602 with 75\%, 25\%, and 5\% of Ae. geniculata (accession TA10437) $5 \mathrm{M}^{\mathrm{g}}$ chromosome, respectively $[43,49]$. Disomic addition line TA7659 was used to develop a

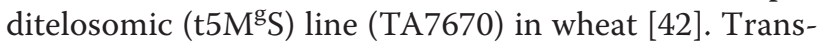
location lines TA5599 [T5DL-5M $\left.{ }^{\mathrm{g}} \mathrm{L} \cdot \mathrm{M}^{\mathrm{g}} \mathrm{S}(0.25)\right]$ and TA5601 [T5DL.5DS-5 $\mathrm{M}^{\mathrm{g}} \mathrm{S}(0.75)$ ] are $\mathrm{BC}_{2} \mathrm{~F}_{5}$ hybrids derived from a disomic substitution line TA6675 [DS5M ${ }^{\mathrm{g}}$ (5D)] and Chinese Spring (CS)Ph ${ }^{I}$ stock [48], followed by backcrossing to the bread wheat cultivar WL711 $[43,49]$. Translocation line TA5602 [T5DL-5DS-5MgS (0.95)] is a $\mathrm{BC}_{3} \mathrm{~F}_{6}$ line derived in a similar way [43]. All the three translocation lines (TA5599, TA5601 and TA5602) carry Lr57 and Yr40 genes. A set of Ae. geniculata accessions (TA2899, TA1800, TA1801, TA2049, TA2786, TA10437 and TA10029) and wheat lines (Chinese Spring, WL711, Jagger and Overley) were also used in this study. Two germplasm lines (KS11WGGRC53-J and KS11WGGR C53-O) released by the WGRC (http://www.k-state.edu/ wgrc/Germplasm/grmplsm.html) were used to validate the $5 M^{g} \mathrm{~S}$ SNPs mapped in TA5602. The germplasm KS11WGGRC53-J was developed by crossing the translocation line TA5602 and winter wheat cultivar Jagger (TA5602/3*Jagger; TA5089-L1) whereas KS11WGGRC53-O was developed by crossing TA5602 with winter wheat cultivar Overley (TA5602/3*Overley; TA5089-L2).

\section{Flow-sorting and next-generation sequencing}

Aqueous suspensions of mitotic metaphase chromosomes were prepared from wheat-Ae. geniculata ditelosomic addition line $5 \mathrm{M}^{\mathrm{g}} \mathrm{S}$ (TA7670) [42] following the protocol of Vrána et al. [58]. The samples were stained by DAPI and analyzed using an FACSAria II SORP flow cytometer and sorter (BD Biosciences, San Jose, USA). Three independent samples of 100,000 chromosomes were sorted into $40 \mu \mathrm{l}$ of sterile deionized water in a $0.5-\mathrm{ml}$ PCR tube. The contamination of sorted fractions by other chromosomes was determined for each sorted run after analyzing 1,000 chromosomes on a microscopic slide. The chromosomes were identified using FISH with probes for the Afa-family and [GAA]n repeats [59]. The DNA 


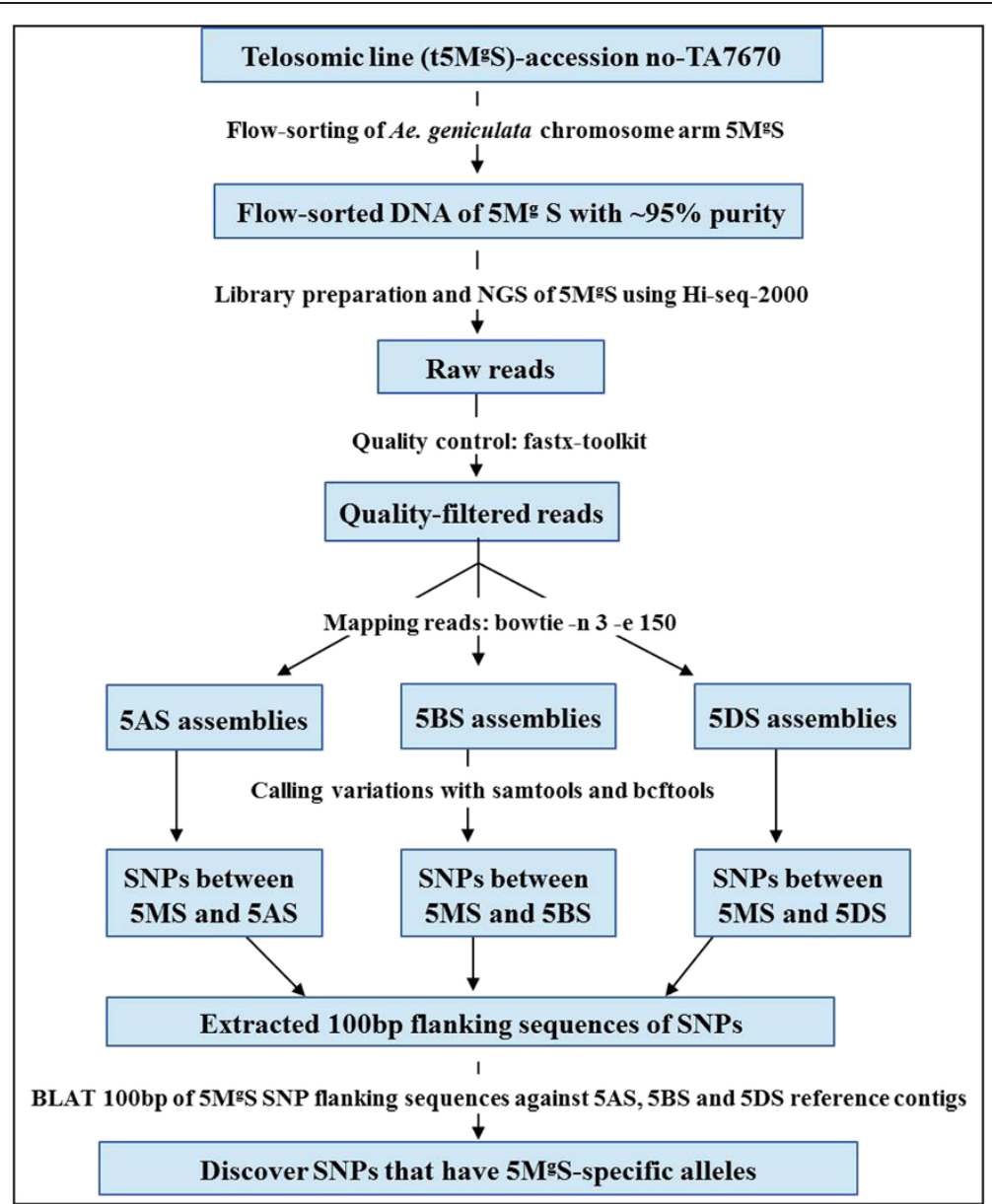

Figure 4 Graphical summary scheme. A schematic showing the strategy used for genome specific SNP discovery for $5 \mathrm{M}^{9} \mathrm{~S}$ of Ae. geniculata.

of chromosomes sorted into PCR tubes was purified and amplified using an Illustra GenomiPhi V2 DNA Amplification Kit (GE Healthcare, Piscataway, USA) as described by Šimková et al. [69]. The three samples of amplified DNA were pooled to reduce possible amplification bias. Sequencing of amplified chromosomal DNA was performed with HiSEq 2000 (Illumina). Pooled MDA-amplified DNA was used to create the corresponding shotgun DNA-seq library. The library for the $5 \mathrm{M}^{\mathrm{g}} \mathrm{S}$ was run in a single lane at DNA core facility services at University of Missouri, USA. For this project we opted for single read sequencing where only one end is sequenced. Sequence data generated from the short arm of Ae. geniculata chromosome arm $5 \mathrm{M}^{g}$ was used for SNP discovery.

\section{Rationale and strategy for read alignment and variant calling}

Before aligning reads to references, all reads were first trimmed to remove the low-quality bases (phred score $<=15$ ) on the end of the reads; reads with more than $30 \mathrm{bp}$ after quality trimming were then filtered with an overall quality ( $80 \%$ of bases must have quality $\geq 15$ ). NGS-based SNP discovery involved two basic steps: (1) alignment of NGS on a reference genome sequence, also called read mapping, and (2) variant calling from the aligned sequences. Variant calling is much easier if a reference sequence is available, because short reads with deep sequencing coverage increases the confidence level for SNP discovery. In this study, we used Chromosome Shotgun Sequences (CSS) as reference sequences and assemblies from wheat chromosome arms 5AS, 5BS and 5DS developed under the International Wheat Genome Sequencing Consortium Survey Sequencing Initiative (http://www.wheatgenome.org/). The $5 \mathrm{M}^{\mathrm{g}} \mathrm{S}$ reads were mapped on these assemblies. In the second step, variants were called against each individual assembly (5AS, 5BS and 5DS). We used the Bowtie software (v1.3) (http://bowtie-bio.sourceforge.net/index.shtml) to map quality filtered reads of $5 \mathrm{M}^{\mathrm{g}}$ to the Chinese Spring $5 \mathrm{~A}$, 5B and 5D contigs separately (Figure 4). The parameters to control the mapping quality were: $-\mathrm{k} 1$-best $-\mathrm{v} 3$. The alignment results were saved in SAM format files. SAMtools (v1.8) (http://samtools.sourceforge.net/) were 
used to generate pileup files, which were then fed to BCFtools (http://samtools.sourceforge.net/) to call raw variations using default parameters. All variations with coverage depth $>=4$, SNP quality $>=50$ were kept for subsequent analysis (Figure 4). We also assembled $5 \mathrm{M}^{\mathrm{g}} \mathrm{S}$ contigs using the SOAPdenovo (http://soap.genomics.org.cn/ soapdenovo.html) to assemble contigs for the $5 \mathrm{M}^{\mathrm{g}}$ genome, with parameter $-\mathrm{k} 27$.

\section{Detection of $\mathrm{M}^{\mathrm{g}}$-genome-specific SNPs}

To identify $5 \mathrm{M}^{\mathrm{g}}$-specific SNPs, the program BLAT was used to align the 100-bp flanking sequences of $5 \mathrm{M}^{\mathrm{g}} \mathrm{S}$ specific SNPs against the 5AS, 5BS and 5DS reference contigs. The alleles of SNPs that had 100\% similarity on the flanking sequences in 5AS, 5BS and 5DS were then compared to select the potential $5 \mathrm{M}^{\mathrm{g}} \mathrm{S}$-specific SNPs.

\section{Validation of SNPs \\ KASPar genotyping based validation}

A set of 44 SNPs were selected for further validation and identification of alien addition and translocation lines. For each putative $5 \mathrm{M}^{\mathrm{g}} \mathrm{S}$-specific SNP, two allele-specific forward primers and one common reverse primer (Additional file 3: Table S1) were designed (KBioscience, Hoddesdon, UK). Genotyping reactions were performed in a final volume of $8.11 \mu \mathrm{l}$, which included a reaction mix: $4.0 \mu \mathrm{l}$ of $2 \mathrm{x}$ reaction mix (per reaction) (KBioscience, Hoddesdon, $\mathrm{UK}$ ), and $0.11 \mathrm{ul}$ assay mix (per reaction) and $\sim 20 \mathrm{ng}$ of genomic DNA $(4.0 \mu \mathrm{L})$. The following cycling conditions were used: $94^{\circ} \mathrm{C}$ for 15 minutes; and 35 cycles of $94^{\circ} \mathrm{C}$ for 10 seconds and $60^{\circ} \mathrm{C}$ for 1 minute, followed by $35^{\circ} \mathrm{C}$ for 30 seconds for plate reading. All reactions used a CFX96 Touch $^{\text {Tw }}$ Real-Time PCR Detection System (Bio-Rad, Hercules, CA, USA), which has an inbuilt fluorescence scanner, and data were analyzed using Bio-Rad CFX manager software under the allelic discrimination mode.

\section{PCR product-based SNP validation}

To assess the veracity of the discovered SNPs and estimate the false-positive SNP discovery rate, 15 sequences, with one SNP each, were randomly chosen from the SNP reference sequences. SNP flanking primers were designed with Primer3 (http://bioinfo.ut.ee/primer3-0.4.0/). Of 15 primers, only 12 primer pairs generated PCR products in both Chinese Spring and Ae. geniculata TA2899. Failure to amplify the target DNA by two primer pairs was due to suboptimal PCR conditions, later confirmed by optimization. The PCR products were eluted from the electrophoresis gels and purified. Purified products were then sequenced with an Applied Biosystems 3730xl DNA Analyzer (Life Technologies, USA) and sequences were aligned and compared for SNPs.

\section{Availability of supporting data}

The $5 \mathrm{M}^{\mathrm{g}}$ short arm sequence data has been submitted to SRA under accession SRX474187. An additional table and two additional figures were also included in the manuscript.

\section{Additional files}

Additional file 1: Figure S1. The frequency and coverage depth of $5 \mathrm{M}^{9} \mathrm{~S}$ assembled sequences.

Additional file 2: Figure S2. Coverage depth of $5 M^{9} S$ sequences against reference contigs 5AS, 5BS and 5DS

Additional file 3: Table S1. List of $1045 \mathrm{M}^{9} \mathrm{~S}$-based, genome-specific SNP markers developed by mapping the $5 \mathrm{M}^{9}$ Illumina reads on 5AS, 5BS and 5DS assemblies of T. aestivum cV. Chinese Spring.

\section{Competing interests}

The authors declare that they do not have any competing interests.

\section{Authors' contributions}

All the listed authors contributed significantly to the manuscript. VKT, JS, SKS and BSG planned the research. BF, BK and PC contributed to the germplasm development used in the experiments. JV, MK and JD carried out chromosome flow-sorting and DNA amplification experiments. VKT, SW, SKS and EA contributed to DNA sequencing, sequence analysis, SNP discovery, validation and introgression mapping experiments. VKT and BSG prepared the manuscript. All authors read and approved the final manuscript.

\section{Acknowledgements}

We thank the International Wheat Genome Sequencing Consortium (Bethesda, USA) for providing pre-publication access to the shotgun sequence assemblies of wheat chromosomes 5AS, 5BS and 5DS. We thank our colleagues Jarmila Číhalíková, Romana Šperková and Zdeňka Dubská for assistance with chromosome sorting and DNA amplification. We thank Jon W. Raupp and Nidhi Rawat, WGRC, for critical reading of the manuscript. JD JV and MK were supported by the Czech Science Foundation (award no. P501/12/G090) and by the Ministry of Education, Youth and Sports of the Czech Republic and the European Regional Development Fund (Operational Programme Research and Development for Innovations No. ED0007/01/01). VKT was supported by a research agreement grant between Kansas State University and King Abdulaziz University. Part of the research in BSG lab was supported by grants from Kansas Wheat Commission and Heartland Plant Innovations. The computing for this project was performed on the Beocat Research Cluster at Kansas State University, which is funded in part by NSF grants CNS-1006860, EPS-1006860, and EPS-0919443. This is the contribution number 14-319-J from Kansas Agricultural Experiment Station, Kansas State University, Manhattan KS 66506-502, USA. Work was done under the auspices of WGRC I/UCRC partially funded by NSF grant contract (IIP-1338897).

\section{Author details}

'Wheat Genetics Resource Center, Department of Plant Pathology, Kansas State University, Manhattan, KS 66506, USA. ²Department of Plant Pathology, Kansas State University, Manhattan, KS 66506, USA. ${ }^{3}$ Institute of Experimental Botany, Centre of the Region Haná for Biotechnological and Agricultural Research, Olomouc CZ-78371, Czech Republic. ${ }^{4}$ School of Agricultural Biotechnology, Punjab Agricultural University, Ludhiana, India. ${ }^{5}$ Department of Biological Sciences, Faculty of Sciences, King Abdulaziz University, Zeddah, Saudi Arabia.

Received: 23 December 2013 Accepted: 31 March 2014 Published: 10 April 2014

\section{References}

1. McFadden ES, Sears ER: The artificial synthesis of Triticum spelta. Rec Genet Soc Am 1944, 13:26-27.

2. Kihara $\mathrm{H}$ : Discovery of the DD-analyzer, one of the ancestors of vulgare wheats. Agric Hortic 1944, 19:889-890 
3. McFadden ES, Sears ER: The origin of Triticum spelta and its free-threshing hexaploid relatives. J Hered 1946, 37:81-89.

4. Qi LL, Friebe B, Zhang P, Gill BS: Homoeologous recombination, chromosome engineering and crop improvement. Chromosome Res 2007, 15:3-19.

5. Ogbonnaya FC, Abdalla O, Mujeeb-Kazi A, Kazi AG, Xu SS, Gosman N, Lagudah ES, Bonnett D, Sorrells ME: Synthetic hexaploid in wheat improvement. In: Janick J (ed). Plant Breeding Rev 2013, 37:35-122.

6. Gill BS, Raupp J: Direct genetic transfers from Aegilops sqaarrosa L. to hexaploid wheat. Crop Sci 1987, 27:445-450.

7. Dvorak J, Luo MC, Yang ZL, Zhang HB: The structure of the Aegilops tauschii genepool and the evolution of hexaploid wheat. Theor Appl Genet 1998, 97:657-670.

8. Roder MS, Korzun V, Wendehake K, Plaschke J, Tixier MH, Leroy P, Ganal MW: A microsatellite map of wheat. Genetics 1998, 149:2007-2023.

9. Qi LL, Echalier B, Chao S, Lazo GR, Butler GE, Anderson OD, Akhunov ED, Dvorák J, Linkiewicz AM, Ratnasiri A, Dubcovsky J, Bermudez-Kandianis CE, Greene RA, Kantety R, La Rota CM, Munkvold JD, Sorrells SF, Sorrells ME, Dilbirligi M, Sidhu D, Erayman M, Randhawa HS, Sandhu D, Bondareva SN, Gill KS, Mahmoud AA, Ma XF, Miftahudin, Gustafson JP, Conley EJ, et al: A chromosome bin map of 16,000 expressed sequence tag loci and distribution of genes among the three genomes of polyploid wheat. Genetics 2004, 168:701-712

10. Olson EL, Rouse MN, Pumphrey MO, Bowden RL, Gill BS, Poland JA: Introgression of stem rust resistance genes SrTA10187 and SrTA10171 from Aegilops tauschii to wheat. Theor Appl Genet 2013, 126(10):2477-2484.

11. Wang J, Luo MC, Chen Z, You FM, Wei Y, Zheng Y, Dvorak J: Aegilops tauschii single nucleotide polymorphisms shed light on the origins of wheat D-genome genetic diversity and pinpoint the geographic origin of hexaploid wheat. New Phytol 2013, 198:925-937.

12. Huang L, Brooks SA, Li W, Fellers JP, Trick HN, Gill BS: Map-based cloning of leaf rust resistance gene $L r 21$ from the large and polyploid genome of bread wheat. Genetics 2003, 164:655-664.

13. Periyannan S, Moore J, Ayliffe M, Bansal U, Wang X, Huang L, Deal K, Luo M-C, Kong X, Bariana H, Mago R, McIntosh R, Dodds P, Dvorak J, Lagudah E: The Gene Sr33, an ortholog of barley Mla Genes, encodes resistance to wheat stem rust race Ug99. Science 2013, 16:786-788.

14. Dvorák J, Zhang HB: Variation in repeated nucleotide sequences sheds light on the phylogeny of the wheat B and $\mathrm{G}$ genomes. Proc Natl Acad Sci U S A 1990, 87:9640-9644.

15. Huang S, Sirikhachornkit A, Su X, Faris J, Gill BS, Heselkorn R, Gorniki P: Genes encoding plastid acetyl-CoA carboxylase and 3-phosphoglycerate kinase of the Triticum/Aegilops complex and the evolutionary history of polyploid wheat. Proc Natl Acad Sci U S A 2002, 99:8133-8138.

16. Akhunov ED, Akhunova AR, Dvorak J: Mechanisms and rates of birth and death of dispersed duplicated genes during the evolution of a multigene family in diploid and tetraploid wheats. Mol Biol Evol 2007, 24:539-550

17. Gill BS, Friebe B, Raupp WJ, Wilson DL, Cox TS, Sears RG, Brown-Guedira GL, Fritz AK: Wheat genetics resource center: the first 25 years. Advan Agron 2006, 89:74-136.

18. Sharma HC, Gill BS: Current status of wide hybridization in wheat. Euphytica 1983, 32:17-31.

19. Jiang J, Friebe B, Gill BS: Recent advances in alien gene transfer in wheat. Euphytica 1994, 73:199-212

20. Friebe B, Jiang J, Raupp WJ, McIntosh RA, Gill BS: Characterization of wheat-alien translocations conferring resistance to diseases and pests: current status. Euphytica 1996, 91:59-87.

21. Sears ER: The transfer of leaf rust resistance from Aegilops umbellulata to wheat. Brookhaven Symp Biol 1956, 9:1-22

22. Mukai Y, Friebe B, Hatchett JH, Yamamoto M, Gill BS: Molecular cytogenetic analysis of radiation-induced wheat-rye terminal and intercalary chromosomal translocations and the detection of rye chromatin specifying resistance to Hessian fly. Chromosoma 1993, 102:88-95.

23. Lukaszewski AJ: Construction of midget chromosomes in wheat. Genome 1997, 40(4):566-569.

24. Friebe B, Zhang P, Linc G, Gill BS: Robertsonian translocations in wheat arise by centric misdivision of univalent at anaphase I and rejoining of broken centromeres during interkinesis of meiosis II. Cytogenet Genome Res 2005, 109:293-297.

25. Lukaszewski AJ, Lapinski B, Rybka K: Limitations of in situ hybridization with total genomic DNA in routine screening for alien introgressions in wheat. Cytogenet Genome Res 2005, 109:373-377.
26. Mullan DJ, Platteter A, Teakle NL, Appels R, Colmer TD, Anderson JM, Francki MG: EST-derived SSR markers from defined regions of the wheat genome to identify Lophopyrum elongatum specific loci. Genome 2006, 48:811-822

27. Akhunov ED, Nicolet C, Dvorak J: Single nucleotide polymorphism genotyping in polyploid wheat with the Illumina GoldenGate assay. Theor Appl Genet 2009, 119:507-517.

28. Allen AM, Barker GL, Berry ST, Coghill JA, Gwilliam R, Allen AM, Barker GL, Berry ST, Coghill JA, Gwilliam R, Kirby S, Robinson P, Brenchley RC, D'Amore R, McKenzie N, Waite D, Hall A, Bevan M, Hall N, Edwards KJ: Transcriptspecific, single-nucleotide polymorphism discovery and linkage analysis in hexaploid bread wheat (Triticum aestivum L.). Plant Biotechnol J 2011, 9:1086-1099.

29. Paux E, Sourdille P, Mackay I, Feuillet C: Sequence-based marker development in wheat: advances and applications to breeding Biotechnol Adv 2011, 30(5):1071-1088.

30. Berkman PJ, Lai K, Lorenc MT, Edwards D: Next generation sequencing applications for wheat crop improvement. Am J Bot 2012, 99:365-371.

31. Cavanagh CR, Chao S, Wang S, Huang BE, Stephen S, Kiani S, Forres K, Saintenac C, Brown-Guedira GL, Akhunova A, See D, Bai G, Pumphrey M, Tomar L, Wong D, Kong S, Reynolds M, da Silva ML, Bockelman H, Talbert L, Anderson JA, Dreisigacker S, Baenziger S, Carter A, Korzun V, Morrell PL, Dubcovsky J, Morell MK, Sorrells ME, Hayden MJ, et al: Genome-wide comparative diversity uncovers multiple targets of selection for improvement in hexaploid wheat landraces and cultivars. Proc Natl Aacad Sci 2013, 110(20):8057-8062.

32. Doležel J, Kubaláková M, Paux E, Bartoš J, Feuillet C: Chromosome-based genomics in the cereals. Chromosome Res 2007, 15:51-66.

33. Doležel J, Vrána J, Šafář J, Bartoš J, Kubaláková M, Šimková H: Chromosomes in the flow to simplify genome analysis. Funct Integr Genomics 2012, 12:397-416.

34. Molnár I, Kubaláková M, Šimková H, Cseh A, Molnár-Láng M, Doležel J: Chromosome isolation by flow sorting in Aegilops umbellulata and $\mathrm{Ae}$. comosa and their allotetraploid hybrids Ae. biuncialis and Ae. geniculata. PLOS ONE 2011, 6(11):e27708.

35. Molnár I, Šimková H, Leverington-Waite M, Goram R, Cseh A, Vrána J, Farkas A Doležel J, Molnár-Láng M, Griffiths S: Syntenic relationships between the U and $\mathrm{M}$ genomes of Aegilops, wheat and the model species Brachypodium and rice as revealed by COS markers. PLOS ONE 2013, 8(8):e70844.

36. Kihara $\mathrm{H}$ : Considerations on the evolution and distribution of Aegilops species based on the analyser method. Cytologia 1954, 19:336-357.

37. Kimber G, Sallee PJ, Feiner MM: The interspecific and evolutionary relationships of Triticum ovatum. Genome 1988, 30:218-221.

38. Gill BS, Sharma HC, Raupp WJ, Browder LE, Hatchett JH, Harvey TL, Mouseman G, Waines JG: Evaluation of Aegilops species for resistance to wheat powdery mildew, wheat leaf rust, Hessian fly, and greenbug Plant Dis 1985, 69:314-316.

39. Zaharieva M, Monneveux P, Henry M, Rivoal R, Valkoun J, Nachit MM: Evaluation of a collection of wild wheat relative Aegilops geniculata Roth and identification of potential sources for useful traits. Euphytica 2001, 119:314-316.

40. Schneider A, Molnar I, Molnar-Lang M: Utilisation of Aegilops (goatgrass) species to widen the genetic diversity of cultivated wheat. Euphytica 2008, 163:1-19.

41. Rawat N, Tiwari VK, Singh N, Randhawa GS, Singh K, Chhuneja P, Dhaliwal HS: Evaluation and utilization of Aegilops and wild Triticum species for enhancing iron and zinc content in wheat. Genet Resour Crop Evol 2009, 56:53-64.

42. Friebe B, Tuleen N, Gill BS: Development and identification of a set of Triticum aestivum-Aegilops geniculata chromosome addition lines. Genome 1999, 42:374-380.

43. Kuraparthy V, Chhuneja P, Dhaliwal HS, Kaur S, Bowden RL, Gill BS: Characterization and mapping of cryptic introgression from $\mathrm{Ae}$. geniculata with new leaf rust and stripe rust resistance genes $L r 57$ and Yr40 in wheat. Theor Appl Genet 2007, 114:1379-1389.

44. Liu W, Rouse M, Friebe B, Jin Y, Gill BS, Pumphrey MO: Discovery and molecular mapping of a new gene conferring resistance to stem rust, Sr53, derived from Aegilops geniculata and characterization of spontaneous translocation stocks with reduced alien chromatin. Chromosome Res 2011, 19:669-682.

45. Okamoto M: Asynaptic effect of chromosome V. Wheat Inf Serv 1957, 5:6. 
46. Sears ER, Okamoto M: Intergenomic chromosome relationships in hexaploid wheat. In Proc X Int Congress Genet. Toronto, Canada: Univ Toronto Press; 1958:258-259.

47. Sears ER: Induced mutant with homoeologous pairing in common wheat. Can J Genet Cytol 1977, 19:585-593.

48. Chen PD, Tsujimoto H, Gill BS: Transfer of $P h /$ genes promoting homoeologous pairing from Triticum speltoides to common wheat. Theor Appl Genet 1994, 88(1):97-101.

49. Aghaee-Sarbarzeh M, Ferrahi M, Singh S, Singh H, Friebe B, Gill BS, Dhaliwal $\mathrm{HS}: \mathrm{Phl}$ induced transfer of leaf and stripe rust resistance genes from Aegilops triuncialis and Ae. geniculata to bread wheat. Euphytica 2002, 127:377-382.

50. Niu ZX, Klindworth DL, Friesen TL, Chao SM, Jin Y, Cai XW, Xu SS: Targeted introgression of a wheat stem rust resistance gene by DNA markerassisted chromosome engineering. Genetics 2011, 187:1011-1021.

51. Liu Z, Li DY, Zhang XY: Genetic relationships among five basic genomes St, E, A, B and D in Triticeae revealed by genomic Southern and in situ hybridization. J Integr Plant Biol 2007, 49:1080-1086.

52. Qi LL, Pumphrey MO, Friebe B, Chen PD, Gill BS: Molecular cytogenetic characterization of alien introgressions with gene Fhb3 for resistance to Fusarium head blight disease of wheat. Theor Appl Genet 2008, 117:1155-1166.

53. Tiwari VK, Rawat N, Singh N, Kumar S, Randhawa GS, Dhaliwal HS: Substitutions of $2 \mathrm{~S}$ and $\mathrm{ZU}$ chromosomes of Aegilops kotschyi in wheat enhance grain iron and zinc concentration. Theor Appl Genet 2010, 121(2):259-269.

54. Peil A, Korzun V, Schubert V, Schumann E, Weber WE, Roder MS: The application of wheat microsatellites to identify disomic Triticum aestivum-Aegilops markgrafii addition lines. Theor Appl Genet 1998, 96:138-146.

55. Nagy ED, Molna'r I, Schneider A, Kova'cs G, Molnár-Láng M: Characterisation of chromosome-specific S-SAP markers and their use to study genetic diversity in Aegilops species. Genome 2006, 49:289-296.

56. Schneider A, Molnár I, Molnár-Láng M: Selection of U and M genome specific wheat SSR markers using wheat-Aegilops biuncialis and wheat-Ae. geniculata addition lines. Euphytica 2010, 175:357-364.

57. Lysák MA, Číhalíková J, Kubaláková M, Šimková H, Künzel G, Doležel J: Flow karyotyping and sorting of mitotic chromosomes of barley (Hordeum vulgare L.). Chromosome Res 1999, 7:431-444.

58. Vrána J, Kubaláková M, Šimková H, Číhalíková J, Lysák MA, Doležel J: Flow-sorting of mitotic chromosomes in common wheat (Triticum aestivum L.). Genetics 2000, 156:2033-2041.

59. Kubaláková M, Valárik M, Bartoš J, Vrána J, Číhalíková J, Doležel J: Analysis and sorting of rye (Secale cereale L.) chromosomes using flow cytometry. Genome 2003, 46:893-905.

60. Kubaláková M, Kovářová P, Suchánková P, Číhalíková J, Bartoš J, Lucretti S, Watanabe N, Kianian S, Doležel J: Chromosome sorting in tetraploid wheat and its potential for genome analysis. Genetics 2005, 170:823-829.

61. Ossowski S, Schneeberger K, Clark RM, Lanz C, Warthmann N, Weigel D: Sequencing of natural strains of Arabidopsis thaliana with short reads. Genome Res 2008, 18:2024-2033.

62. Yamamoto T, Nagasaki H, Yonemaru J, Ebana K, Nakajima M, Shibaya T, Yano M: Fine definition of the pedigree haplotypes of closely related rice cultivars by means of genomewide discovery of single-nucleotide polymorphisms. BMC Genomics 2010, 11:267.

63. Trebbi D, Maccaferri M, de Heer P, Sørensen A, Giuliani S, Salvi S, Sanguineti MC, Massi A, van der Vossen EAG, Tuberosa R: High-throughput SNP discovery and genotyping in durum wheat (Triticum durum Desf.). Theor Appl Genet 2011, 123:555-569.

64. Close TJ, Bhat PR, Lonardi S, Wu Y, Rostoks N, Ramsay L, Druka A, Stein N, Svensson JT, Wanamaker S, Bozdag S, Roose ML, Moscou MJ, Chao S, Varshney RK, Szucs P, Sato K, Hayes PM, Matthews DE, Kleinhofs A Muehlbauer GJ, DeYoung J, Marshall DF, Madishetty K, Fenton RD, Condamine P, Graner A, Waugh R: Development and implementation of high-throughput SNP genotyping in barley. BMC Genomics 2009, 10:582.

65. Waugh $R$, Jannink JL, Muehlbauer GJ, Ramsay L: The emergence of whole genome association scans in barley. Curr Opin Plant Biol 2009, 12:218-222.

66. Treangen TJ, Salzberg SL: Repetitive DNA and next-generation sequencing: computational challenges and solutions. Nat Rev Genet 2012, $13: 36-46$
67. Trick M, Adamski NM, Mugford SG, Jiang C-C, Febrer M, Uauy C: Combining SNP discovery from next-generation sequencing data with bulked segregant analysis (BSA) to fine-map genes in polyploid wheat. BMC Plant Biol 2012, 12:14.

68. Ravel C, Praud S, Murigneux A, Canaguier A, Sapet F, Samson D, Balfourier F, Dufour P, Chalhoub B, Brunel D, Beckert M, Charmet G: Single-nucleotide polymorphism frequency in a set of selected lines of bread wheat (Triticum aestivum L.). Genome 2006, 49:1131-1139.

69. Šimková H, Svensson JT, Condamine $P$, Hřibova' E, Suchánková $P$, Bhat $P$, Bartoš J, Šafár J, Close T, Doležel J: Coupling amplified DNA from flow-sorted chromosomes to high-density SNP mapping in barley. BMC Genomics 2008, 9:294-299.

doi:10.1186/1471-2164-15-273

Cite this article as: Tiwari et al:: SNP Discovery for mapping alien introgressions in wheat. BMC Genomics 2014 15:273.

\section{Submit your next manuscript to BioMed Central and take full advantage of:}

- Convenient online submission

- Thorough peer review

- No space constraints or color figure charges

- Immediate publication on acceptance

- Inclusion in PubMed, CAS, Scopus and Google Scholar

- Research which is freely available for redistribution 